ARTICLE

Received 4 Feb 2014 | Accepted 30 Jul 2014 | Published 26 Sep 2014

\title{
Arabidopsis cell expansion is controlled by a photothermal switch
}

Henrik Johansson ${ }^{1, \star, \dagger}$, Harriet J. Jones ${ }^{1, \star}$, Julia Foreman ${ }^{1}$, Joseph R. Hemsted ${ }^{1}$, Kelly Stewart ${ }^{1}$, Ramon Grima ${ }^{1} \&$ Karen J. Halliday ${ }^{1}$

In Arabidopsis, the seedling hypocotyl has emerged as an exemplar model system to study light and temperature control of cell expansion. Light sensitivity of this organ is epitomized in the fluence rate response where suppression of hypocotyl elongation increases incrementally with light intensity. This finely calibrated response is controlled by the photoreceptor, phytochrome $\mathrm{B}$, through the deactivation and proteolytic destruction of phytochromeinteracting factors (PIFs). Here we show that this classical light response is strictly temperature dependent: a shift in temperature induces a dramatic reversal of response from inhibition to promotion of hypocotyl elongation by light. Applying an integrated experimental and mathematical modelling approach, we show how light and temperature coaction in the circuitry drives a molecular switch in PIF activity and control of cell expansion. This work provides a paradigm to understand the importance of signal convergence in evoking different or non-intuitive alterations in molecular signalling.

\footnotetext{
${ }^{1}$ Synthetic and Systems Biology (SynthSys), University of Edinburgh, CH Waddington Building, Mayfield Road, Edinburgh EH9 3JD, UK. ${ }^{\star}$ These authors contributed equally to this work. † Present address: Institute of Biology/Applied Genetics, Dahlem Centre of Plant Sciences (DCPS), Freie Universität Berlin, Albrecht-Thaer-Weg 6, D-14195 Berlin, Germany. Correspondence and requests for materials should be addressed to K.J.H.

(email: karen.halliday@ed.ac.uk).
} 
T he light-triggered conversion from skotomorphogenic to photomorphogenic growth (de-etiolation) is a vital developmental step in the early plant life cycle. Seedling de-etiolation results in the opening and expansion of the cotyledons, unfolding of the apical hook and inhibition of hypocotyl elongation ${ }^{1}$. The previous decades of research have identified multiple photoreceptors, downstream factors and hormonal pathways regulating and fine tuning this process ${ }^{2-5}$, often utilizing seedling hypocotyl length to measure the extent of de-etiolation. As elongation of this simple organ is also regulated by ambient temperature, the hypocotyl provides a tractable system to study the intersection of light and temperature ${ }^{6-11}$.

The red light photoreceptor, phytochrome $\mathrm{B}$ (phyB) is exquisitely tuned to detect changes in the ambient light environment. Synthesized in its inactive $\operatorname{Pr}$ form, phyB is photoconverted to active Pfr following exposure to red light, while far-red light reverses this process. Pr to Pfr conversion triggers cytosolic to nuclear localization and aggregation in subnuclear foci or speckles ${ }^{12}$. Pfr is also subject to 'dark reversion', or thermal relaxation to the ground $\operatorname{Pr}$ state, independently of light ${ }^{13}$. The photoreversibility of phyB delivers dynamic light quality sensing, particularly attuned to detect far-red-rich environments that signify potential crowding from neighbouring plants. However, phyB is also a reliable sensor of light quantity. This is exemplified in the much studied fluence rate response curve, where phyB suppression of hypocotyl cell expansion increases with red light fluence rate ${ }^{14,15}$. A recent kinetic model of phyB action elucidated the molecular properties of phyB required for light intensity sensing ${ }^{14}$. This study showed that the photochemical reactions alone were insufficient to deliver the fluence rate response, and in fact dark reversion and nuclear speckling were important dynamic processes in this response. Modelling approaches have also been invaluable in deciphering the highly complex phyA signal transduction ${ }^{16}$.

Hypocotyl elongation is under the dual control of phyB and the phytochrome-interacting factors (PIFs) bHLH transcription factors. PIFs are potent promoters of hypocotyl extension, and phyB constrains PIF action by triggering PIF degradation by the proteasome and through direct sequestration preventing PIF binding to target promoters ${ }^{17,18}$. Conversely, PIFs induce constitutive photomorphogenic 1 (COP1) E3 ligase-dependent proteolysis of phyB $^{19}$. However, we do not yet understand how this dual-negative regulation impacts signalling and the end point physiological responses.

While phyB action represses hypocotyl elongation, warm temperature promotes it. Several studies have shown that PIF4, and to a lesser extent PIF5 stimulate hypocotyl extension in response to heat ${ }^{5,7,10,11,20-21}$. Thus, PIF4 and PIF5 operate at the intersection of light and temperature signalling. High temperature promotes PIF4 binding to the promoters of genes that control biosynthesis of the plant hormone auxin, which in turn promotes elongation ${ }^{7,8,11}$. Interestingly, an earlier report showed that light is required for the auxin-induced hypocotyl cell elongation at high temperatures ${ }^{8}$. This observation is in apparent conflict with current understanding that light, acting through photoreceptors such as phyB, is a strong suppressor of hypocotyl extension.

The bZIP transcription factor long-hypocotyl 5 (HY5) acts in opposition to PIFs. This antagonism is evident in loss-of-function mutants: hy5 has a long-hypocotyl phenotype, while pif mutants are shorter than wild type ${ }^{22,23}$. Like PIFs, HY5 protein levels are regulated by light, but unlike PIFs that are degraded, light promotes HY5 protein accumulation. Here light acts in part by reducing the nuclear levels of the E3 ubiquitin ligase component COP1, which targets HY5 for proteasome-mediated degradation $^{24,25}$. HY5 and PIFs are known to share a broad suite of gene targets and recently they have been shown to elicit opposing action through collocation at gene promoters ${ }^{26,27}$. In common with PIF4, HY5 participates in temperature signalling but, while PIF4 operates at higher temperatures, HY5 is an important regulator of cold signalling, promoting anthocyanin accumulation ${ }^{20,28,29}$.

In this study, using the hypocotyl elongation response as a physiological readout, we aimed to gain a systems level understanding of how changes in the light and temperature environment impact signalling through the phyB-PIF motif. Here we report that the 'classical' hypocotyl response to fluence rate is temperature dependent, exposing a gap in our fundamental understanding of light signal transduction. To investigate this, we developed the first phyB signalling model that incorporates phyB-PIF dual-negative feedback regulation. Our data show that phyB action does not saturate at relatively low fluence rates, as previously thought, but continues to inhibit hypocotyl elongation in high light. At cooler temperatures, this high fluence rate response requires the action of HY5. By analysing the phyB-PIF signalling module at a systems level, we solve the dilemma of how light, which is a potent repressor of hypocotyl extension, can switch to a promoter in the warm.

\section{Results}

Red light promotes hypocotyl extension at high temperature. A plethora of literature supports the long-held view that hypocotyl extension is progressively suppressed by increasing red light fluence rates ${ }^{30}$. While we observe this standard response at $17^{\circ} \mathrm{C}$ and $22^{\circ} \mathrm{C}$ (Fig. 1a and Supplementary Fig. 1a), at $27^{\circ} \mathrm{C}$, above a low-light threshold $\left(\sim 1 \mu \mathrm{mol} \mathrm{m}^{-2} \mathrm{~s}^{-1}\right)$, elevated fluence rates incrementally promote elongation. This finding holds true for multiple Arabidopsis accessions (Supplementary Fig. 1b) illustrating that across ecotypes heat induces a complete response inversion from light inhibition to light promotion of hypocotyl extension. Our data illustrate that light and temperature do not act in isolation; rather, the convergence of these signals drives a 'photothermal switch' in response.

As PIF4 and PIF5 have also been implicated in temperaturedependent hypocotyl elongation, they presented good candidate regulators of the photothermal switch $6,10,20,31$. In fact, sequential removal of PIF4, PIF5 and PIF3 led to a gradual suppression of hypocotyl length at both $17^{\circ} \mathrm{C}$ and $27^{\circ} \mathrm{C}$ and importantly, abolished the fluence rate-driven hypocotyl extension at $27^{\circ} \mathrm{C}$ (Fig. 1b,c). Previous studies in white light have shown that PIF4 regulates hypocotyl extension in a temperature-dependent manner ${ }^{10,20}$. Our data demonstrate that in red light, PIF4 operates over a temperature range. Moreover, the large impact of pif4 at both temperatures and the complete elimination of lightinduced hypocotyl extension at $27^{\circ} \mathrm{C}$, indicate that PIF4 has a prominent role in the photothermal switch.

At $27^{\circ} \mathrm{C}$ hypocotyl length does not correlate with PIF levels. As biological reactions are temperature dependent, we postulated that at $27^{\circ} \mathrm{C}$ phyB signalling may become less efficient at higher fluence rates due to increased rate of photoconversion between $\mathrm{Pr}$ and Pfr. This scenario predicts that at $27^{\circ} \mathrm{C}$ phyB would be less effective at degrading PIF proteins at increased fluence rates. However, we observe a strong fluence rate-dependent depletion of PIF4-HA, PIF4-LUC and PIF3-LUC at both temperatures (Fig. 2a,b and Supplementary Fig. 1c). It therefore appears that phyB regulation of PIF protein levels is not compromised at high temperatures. As the PIF constructs we used in this study were all driven by the $35 \mathrm{~S}$ promoter, we wanted to establish whether control was delivered at the transcript level. While we did observe the previously reported light and temperature dependence of PIF4 and PIF5 transcript abundance ${ }^{10,32,33}$, levels did not correlate 

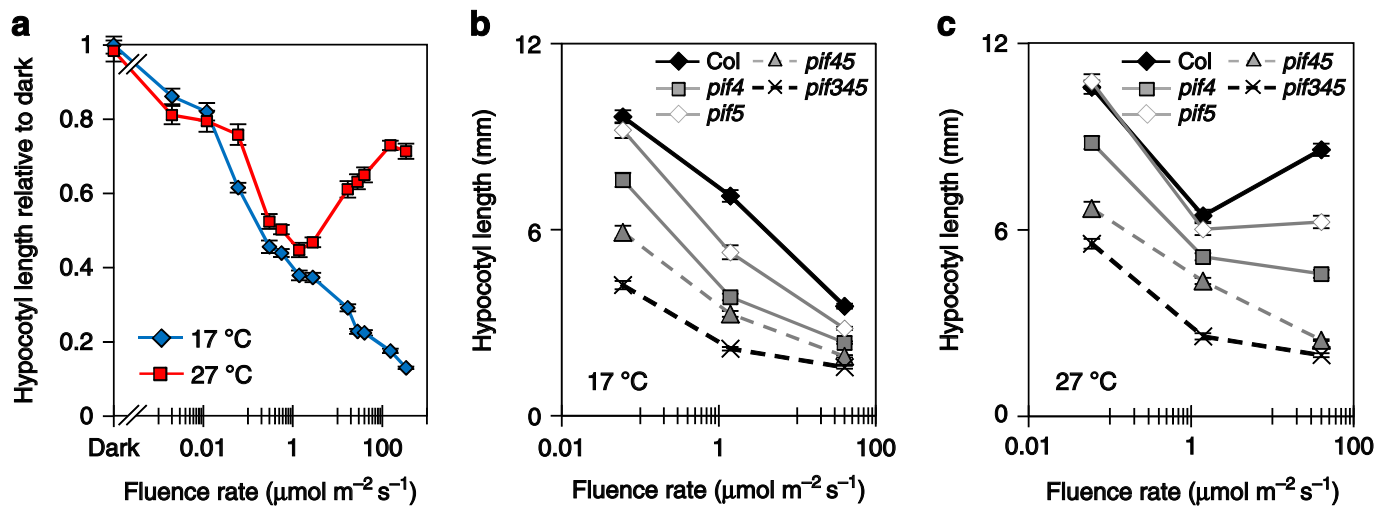

Figure 1 | The fluence rate-dependent switch in hypocotyl regulation at warm temperatures requires PIFs. (a) Fluence rate response curves measuring hypocotyl elongation of 7-day-old WT seedlings grown in continuous red light at $17{ }^{\circ} \mathrm{C}$ and $27^{\circ} \mathrm{C}$ shown as relative to dark at $17{ }^{\circ} \mathrm{C}$. Sample number $>17$, error bars represent s.e.m. (b,c) Fluence rate response curves measuring hypocotyl length of WT, pif4-101, pif5-3, pif4-101pif5-3 and pif3-3pif4-2pif5-3 at $17^{\circ} \mathrm{C}$ (b) and $27^{\circ} \mathrm{C}$ (c) grown at indicated fluence rate of red light. Sample number $>16$, error bars represent s.e.m.

with the hypocotyl response at either $17^{\circ} \mathrm{C}$ or $27^{\circ} \mathrm{C}$ (Fig. 2c,d). These data suggest that phyB remains active at $27^{\circ} \mathrm{C}$ and, as a consequence, PIF levels inversely correlate with fluence rate. PhyB depletion of PIF levels is thought to underlie the classical fluence rate-dependent suppression of hypocotyl elongation, yet at $27^{\circ} \mathrm{C}$, we observe the converse.

Development of the phyB-PIF model. To help us understand the light- and temperature-induced changes that give rise to this altered signalling through the phyB-PIF circuitry, we adopted a mathematical modelling approach. The Rausenberger et al. ${ }^{14}$ phyB model incorporates early events of phyB signalling including dimerization, light-dependent $\mathrm{Pr}$ to Pfr transformation, Pfr to Pr dark reversion, translocation from cytoplasm to nucleus, formation of nuclear speckles and light-dependent degradation ${ }^{13,14,19}$. We extended this model to incorporate the mutual proteolytic destruction of phyB and PIF (following phyB Pfr-PIF interaction) and PIF control of hypocotyl length; we refer to this as Model I (Supplementary Fig. 2a). Similar to the Rausenberger model, Model I can simulate phyB-controlled fluence rate-dependent hypocotyl inhibition (Fig. 3a).

The model predicts $\mathrm{X}$ is required for phyB action. In Model I (and the Rausenberger phyB model), the phyB-induced fluence response saturates at low fluence rates predicted to achieve maximum photoconversion to active Pfr. In contrast, our $17^{\circ} \mathrm{C}$ data show that the hypocotyl inhibition response continues into the higher fluence rate range (Fig. 3a). This discrepancy identifies a gap in our understanding of light control of this response. In Model I, hypocotyl elongation is directly linked to PIF levels (Supplementary Methods). Therefore, further suppression of hypocotyl elongation inhibition at higher fluence rates could in principle, be induced by a concomitant rise in phyB and fall in PIF levels. To test this, phyB and PIF3 protein (that is acutely sensitive to phyB levels) were measured over an extended fluence rate range. Our data illustrate that both proteins deplete to a stable low level at $17^{\circ} \mathrm{C}$ and at $27^{\circ} \mathrm{C}$ (Supplementary Figs $1 \mathrm{c}$ and $3 \mathrm{a}$ ). Based on this finding, we postulated the existence of a new fluence rate component $\mathrm{X}$ which represses PIF activity, particularly at higher fluence rates (see Model II in Supplementary Methods; Supplementary Fig. 2c). With this model modification, we were indeed able to match the experimental data: compare dashed line (Model I), with solid line (Model II) (Fig. 3a). As we observed hypocotyl elongation rather than inhibition at $27^{\circ} \mathrm{C}$, we surmised that, as well as fluence rate dependent, $\mathrm{X}$ may be temperature dependent, with greater potency at cooler temperatures.
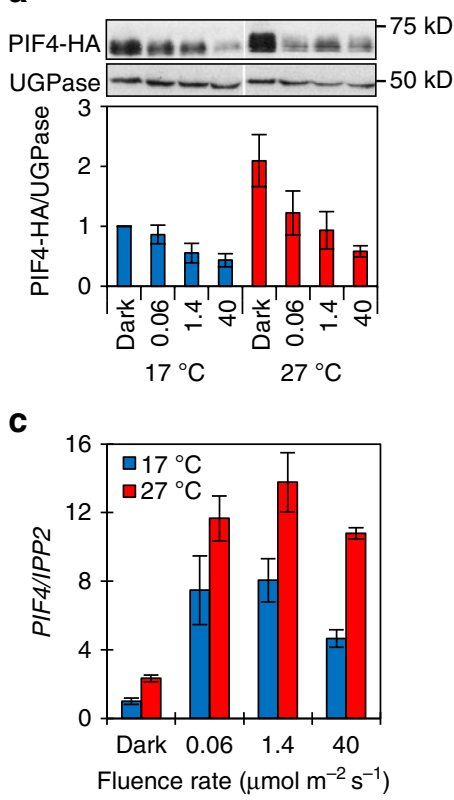

b

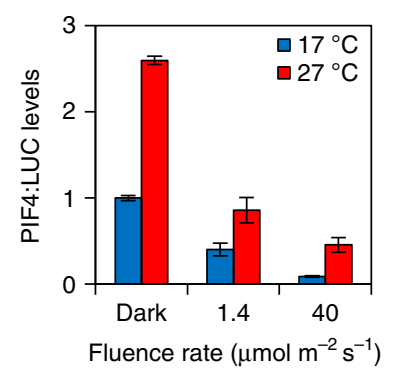

d

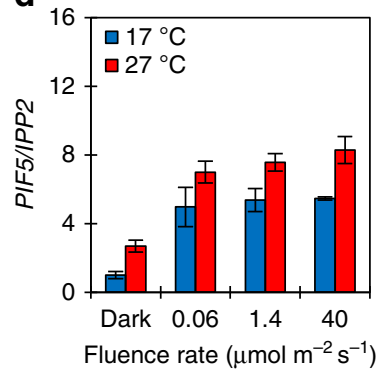

Figure 2 | PIF protein and transcript levels do not correlate with activity. (a) Western blot of 6-day-old 35S:::PIF4-HA seedlings grown at indicated temperature and continuous fluence rate of red light using an HA-specific antibody for detection of PIF4-HA and anti-UGPase as loading control

(upper panel). Quantification of PIF4-HA protein levels relative to UGPase (lower panel). Error bars represent s.e.m., $n=3$. (b) PIF4-LUC levels relative to total protein in 6-day-old seedlings grown at indicated temperatures and fluence rates of red light. Error bars represent s.e.m., $n=3$. Quantification of PIF4 (c) and PIF5 (d) transcript levels relative to IPP2 in seedlings grown as in a. Error bars represent s.e.m., $n=3$.

Experimental validation of HY5 as a component of X. Several antagonists of PIF action have been reported $2,34,35$, however, in this category, the bZIP transcription factor HY5 is a good candidate for $\mathrm{X}$ as it is known to be regulated by both light and temperature ${ }^{24,28}$. A stipulation of Model II is that $\mathrm{X}$ action increases with fluence rate. Our data show that the previously reported hy5 long-hypocotyl phenotype and the accumulation of HY5 protein are both strictly fluence rate dependent in red light (Fig. 3b,c) $)^{22}$. HY5 therefore fulfils the functional requirements for $\mathrm{X}$ in our model and likely constitutes a major component of X. Like HY5, HFR1 and DELLAs are well-known suppressors 

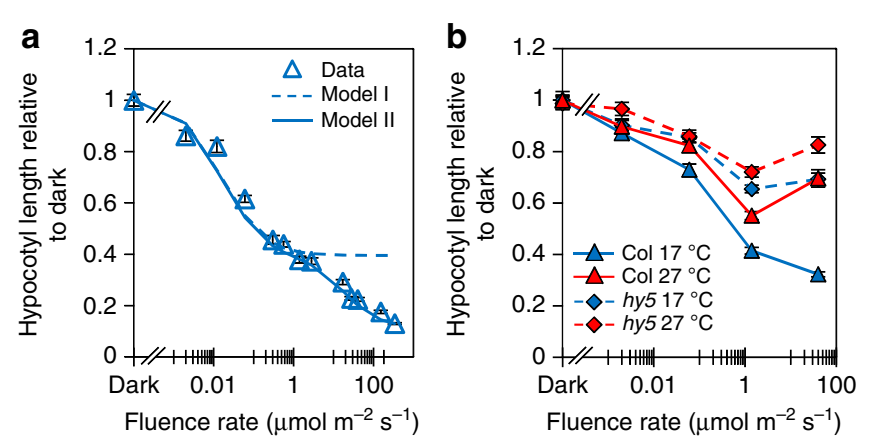

C

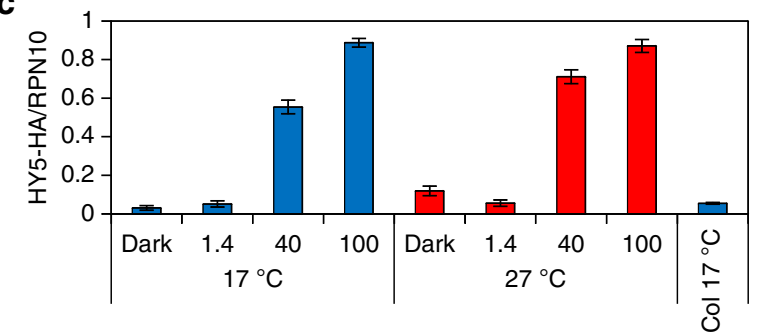

d

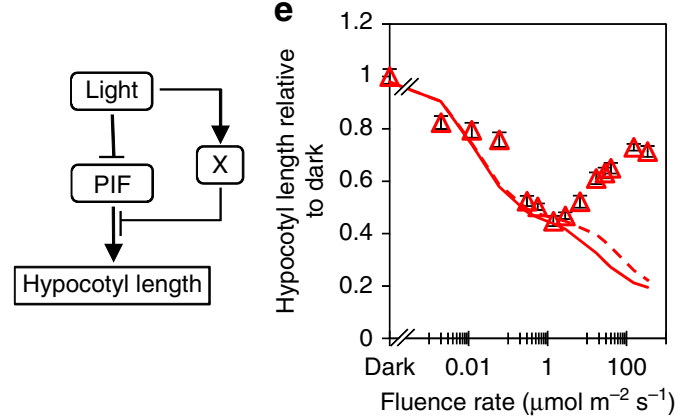

Figure 3 | Suppression of PIF activity by $\mathbf{X}$ is required to repress hypocotyl elongation at high fluence rates. (a) Comparison of $17{ }^{\circ} \mathrm{C}$ experimental data with model I and model II simulation. (b) Fluence rate response curve measuring hypocotyl elongation of 7-day-old WT and hy5 seedlings grown under continuous red light at $17^{\circ} \mathrm{C}$ and $27^{\circ} \mathrm{C}$. Sample number $>20$, error bars represent s.e.m. (c) Quantification of HY5-HA protein levels relative to RPN10 in seedlings grown at indicated temperatures and fluence rates. Error bars represent s.e.m., $n=3$. (d) Diagram of coherent feed-forward motif at $17^{\circ} \mathrm{C}$. (e) Temperature dependency of Model II. Experimental data at $27^{\circ} \mathrm{C}$ (red triangles), simulation data at $27^{\circ} \mathrm{C}$ with temperature dependency ( $\mathrm{X}$, red solid line: $\mathrm{X}$ and all other temperature-dependent parameters, red-dashed line).

of PIF action, we therefore tested whether they could contribute to $\mathrm{X}^{2,3,26,27,34}$. In line with previous observations, we did not observe a marked $h f r 1$ hypocotyl phenotype in our red light conditions $^{36-38}$ (Supplementary Fig. 4a). In contrast, at $17^{\circ} \mathrm{C}$ and $27^{\circ} \mathrm{C}$, we did observe a subtle increase in the della4 ( rgll-1;rgl21;gai-6;rga-2) mutant hypocotyl length compared with the wild type at the higher $40 \mu \mathrm{mol} \mathrm{m}^{-2} \mathrm{~s}^{-1}$ fluence rate (Supplementary Fig. 4b). This indicates that DELLAs may contribute to X, but in a more minor way. We also speculated that HY5 action may be temperature dependent. Figure $3 c$ shows that in continuous red light, HY5 protein levels are not altered by temperature in our experimental range, but the impact of hy5-215 is more influential at $17^{\circ} \mathrm{C}$ than $27^{\circ} \mathrm{C}$, suggesting that HY5 is indeed more active at cooler temperatures (Fig. 3b). Our data therefore validate the necessity for $\mathrm{X}$. It also suggests that at moderate temperatures, hypocotyl elongation is regulated by a coherent feed-forward motif where levels and activity of PIFs are repressed by light (Fig. 3d). Simulated data obtained from Model II illustrates that failure of either motif arm leads to incomplete hypocotyl inhibition (Supplementary Fig. 5).

Development of Model III, the phyB-PIF photothermal model. To gain a more thorough understanding of how temperature impacts signalling through the phyB-PIF network, we incorporated temperature regulation into Model II by assigning $\mathrm{Q}_{10}$ values to specific parameters (Supplementary Methods). We observe a modest temperature-induced rise in phyB levels in dark grown seedlings (Supplementary Fig. 3a) and therefore assigned Pr formation rate, $\left(n_{1}\right)$ mildly temperature sensitive. Likewise, fluence rate depletion of phyB (by PIFs) and PIF4/PIF3 (by phyB) is moderately temperature dependent, identifying $\mathrm{m}_{3}$ (PIFdependent degradation of Pfr) and $\mathrm{m}_{4}$ (Pfr-dependent degradation of PIF) as temperature parameters (Fig. 1d; Supplementary Figs $1 \mathrm{c}$ and $3 \mathrm{a}$ and Supplementary Methods). PIF4 formation rate $\left(n_{2}\right)$, dark reversion rate $\left(p_{1}\right)$ and the basal rate of cell expansion ( $\alpha$ in the model), are reported to be temperature dependent ${ }^{6,14,20}$. Finally, our genetic data (Fig. 3b) suggested the activity of $\mathrm{X}$ (HY5) $b_{1}$, is also temperature dependent. Interestingly, a detailed sensitivity analysis identified a large subset of these parameters $\left(n_{1}, n_{2}, m_{3}, m_{4}\right.$ and $\left.\alpha\right)$ as those causing, on perturbation, a significant change in light-dependent hypocotyl elongation. The analysis also identified $g_{1}$ (Michaelis-Menten constant) as a further sensitive parameter (Supplementary Fig. 6; Supplementary Table 1 and Supplementary Methods).

' $\mathrm{X}$ ' cannot generate the photothermal switch. As we have shown that X (HY5) strongly antagonizes PIF action at $17^{\circ} \mathrm{C}$, but not at $27^{\circ} \mathrm{C}$, we were intrigued to establish whether $\mathrm{X}$ could generate the temperature-activated switch in behaviour. The model, however, could not generate the observed fluence rate-dependent increase in hypocotyl length at $27^{\circ} \mathrm{C}$, suggesting $\mathrm{X}$ action does not drive the switch (Fig. 3e). A global parameter search illustrated that over an extensive range of possible $Q_{10}$ values, Model II cannot recapitulate the switch (Supplementary Methods). These limitations of the model at $27^{\circ} \mathrm{C}$ indicated that additional components were required to elicit the switch in light control of elongation.

Light promotes PIF action at $27^{\circ} \mathrm{C}$. When we analysed data from seedlings expressing 35S:PIF4-LUC or 35S:PIF5-HA, we noted that hypocotyl elongation is suppressed at higher fluence rates at $17^{\circ} \mathrm{C}$ but not at $27^{\circ} \mathrm{C}$ (Fig. 4a,b). Furthermore in the 35S:PIF5-HA seedlings, we observed a fluence rate-dependent rise in hypocotyl extension. This provided additional support for the inclusion of a light-regulated PIF suppressor (X) that operates in the cool, but also suggested PIF action may be boosted by light at $27^{\circ} \mathrm{C}$. As light is thought to solely act as a negative regulator of PIFs, this proposition is at apparent odds with current understanding. To test this hypothesis, we introduced a second fluence rate-dependent component $\mathrm{Y}$, which promotes PIF activity in a temperature-dependent manner (Fig. 4c,d and Model III, Supplementary Methods). With the inclusion of Y, Model III can fully replicate the photothermal switch, which supports our hypothesis, and suggests that the convergence of light and temperature on PIF activity underlies the response inversion.

To test this proposition, we examined the impact of light and temperature on PIF activity by measuring transcript levels of HFR1 and XTR7, two direct targets of PIF4 and PIF5 (ref. 34). Contrary to our expectations, we observed that at both temperatures, HFR1 and XTR7 levels fell in a fluence rate-dependent manner (Fig. 5a), suggesting that these genes do not participate in the temperature-activated switch. In contrast, levels of IAA19, IAA29 and ATHB2 (direct PIF4 targets) and 
a

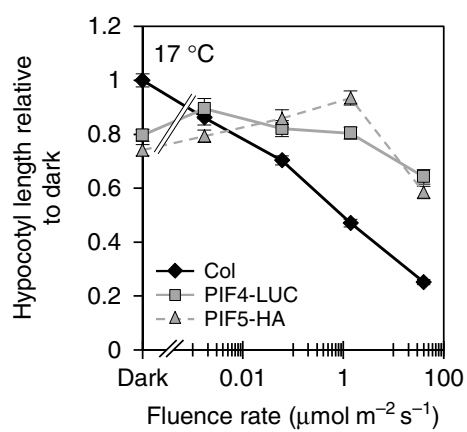

b

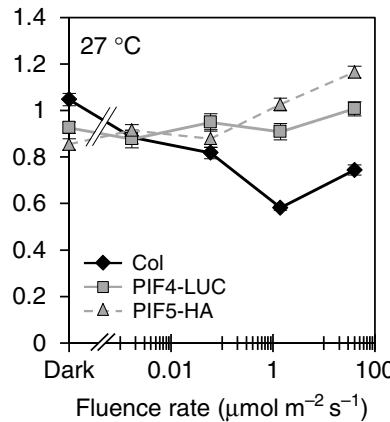

C

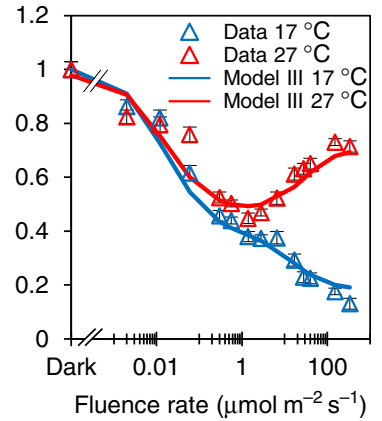

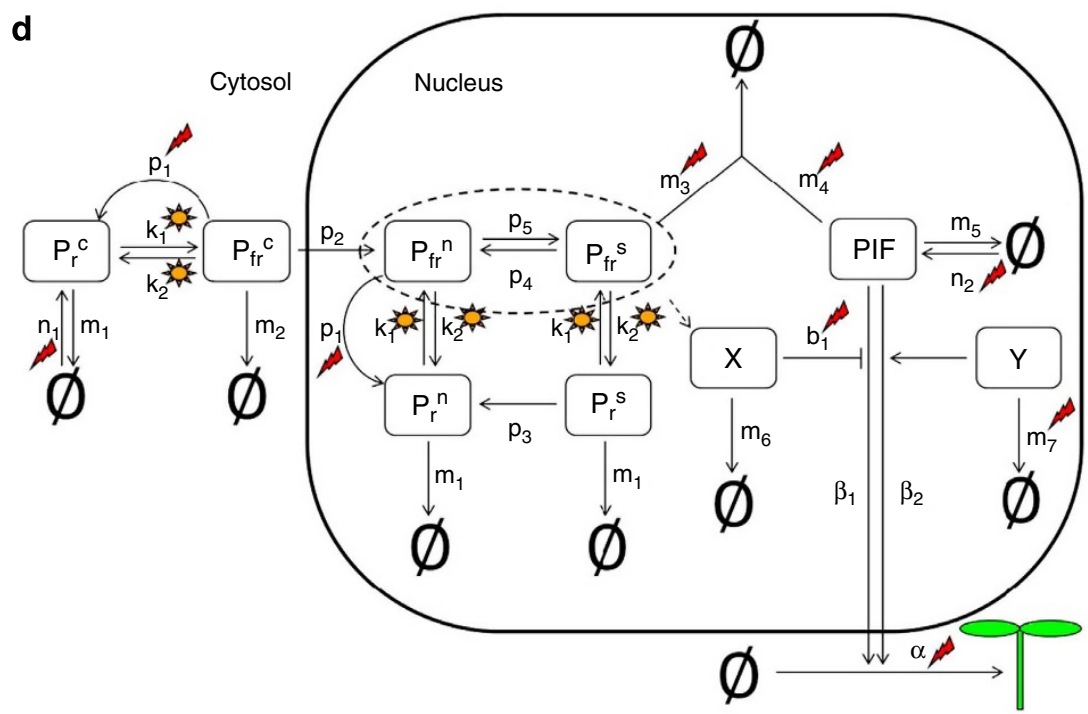

Figure 4 | Model III predicts that light promotes PIF activity at $27^{\circ} \mathbf{C} .(\mathbf{a}, \mathbf{b})$ Hypocotyl measurements of 7-day-old WT, overexpressing lines of PIF4-LUC and PIF5-HA in $17^{\circ} \mathrm{C}$ (a) and $27^{\circ} \mathrm{C}$ (b) grown at indicated fluence rate of continuous red light and shown as relative to the dark value of WT at $17^{\circ} \mathrm{C}$. Sample number $>18$, error bars represents s.e.m. (c) Experimental data and simulated data from Model III of hypocotyl elongation at indicated fluence rates of red light and temperature. (d) A schematic representation of Model III including factors $\mathrm{X}$ and $\mathrm{Y}$. $\mathrm{X}$ can be modelled as either phyB dependent or phyB independent. Suns and flashes represent light- and temperature-regulated events, respectively.

SAUR23, auxin signalling genes previously implicated in PIF4regulated temperature responses, did correlate with the hypocotyl phenotype; depleting with fluence rate in the cool and rising in the warm (Fig. 5b) $)^{7,11,20}$. Application of the auxin transport inhibitor NPA completely blocked the switch, preventing lightactivated hypocotyl elongation at $27^{\circ} \mathrm{C}$, illustrating the prominent role of the auxin pathway in this response (Fig. 5c). Our results are congruent with the recent finding that heat activates PIF4 binding to target promoters by displacing H2A.Znucleosomes that would otherwise block PIF4 access 9 . However, we show that the thermal promotion of PIF action is entirely light dependent. Our data therefore validate the model prediction that the photothermal switch is mediated by complete transformation in light control of PIF target genes that are repressed at $17^{\circ} \mathrm{C}$, but activated at $27^{\circ} \mathrm{C}$. Our data also suggest the switch is controlled by a specific gene set that include auxin pathway genes. This view was further strengthened by our finding that HY5 loss led to a de-repression of IAA19 and IAA29 mRNA levels, at $17^{\circ} \mathrm{C}$, particularly at higher fluence rates, but had little effect at $27^{\circ} \mathrm{C}$ (Fig. 5d). The finding concurs with the recent work showing that HY5 directly opposes PIF action through direct targeting of common genes 26,27 .
Model III predicts continuous light irradiance is required for the switch. Our refined model (Model III) recapitulates experimental data obtained under constant illumination by postulating two fluence rate and temperature-dependent components, $\mathrm{X}$ and Y. To further test the model's predictive power, we considered the case where fluence is delivered in 5- or 15-min pulses that deliver total fluence that matches continuous irradiance controls. The model predicts that under such pulsed light conditions, the switch from inhibition to promotion of hypocotyl extension is not elicited. In experimental pulsed conditions, we observed a modest hypocotyl inhibition response at $17^{\circ} \mathrm{C}$, but these conditions did not generate the switch to elongation at $27^{\circ} \mathrm{C}$ (Supplementary Fig. 8c,d). Thus, constant illumination does indeed appear to be required for the temperature-dependent switch. In the model the rate of production of $\mathrm{X}$ and $\mathrm{Y}$ is directly proportional to the light intensity. This can be achieved in different ways, for example, if the rate of X/Y production is proportional to the flux from Pr to Pfr, or to a photosynthetic output. Our model cannot discriminate between these alternative light input scenarios. However, this analysis confirms the model's utility as a reliable tool to predict growth under varying light conditions such as those found in the natural environment. 

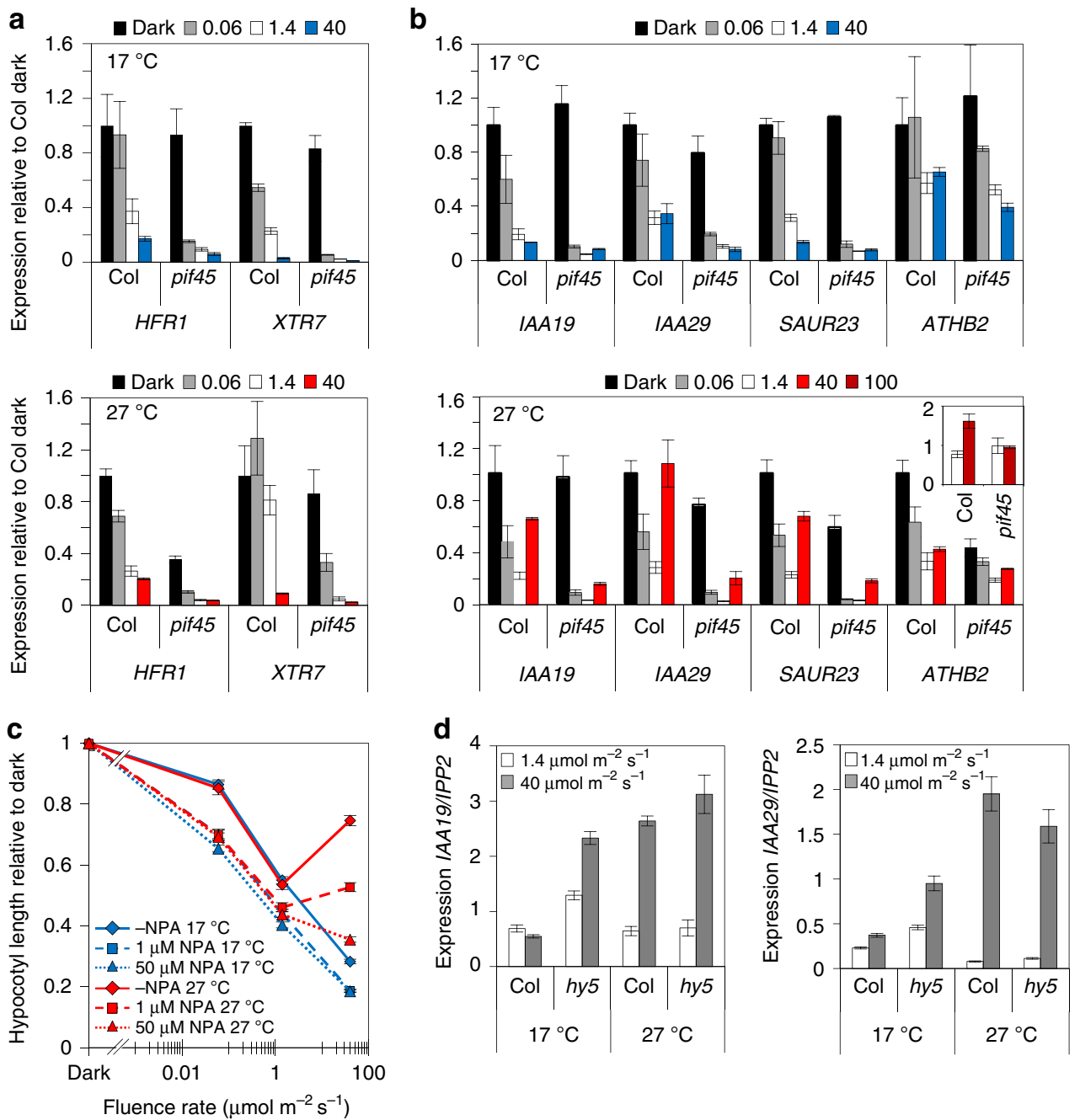

Figure 5 | At $27^{\circ} \mathbf{C}$, PIF4/5-dependent transcriptional regulation of auxin targets is promoted at high fluence rates. (a,b) Transcript levels of HFRT, XTR7, IAA19, IAA29, SAUR23 and ATHB2 relative to IPP2 in 6-day-old WT and pif4-101pif5-3 seedlings grown in dark, $0.06,1.4$ or $40 \mu \mathrm{mol} \mathrm{m}^{-2} \mathrm{~s}^{-1}$ of red light at indicated temperature. Insert: expression of $A T H B 2$ in WT and pif4-101pif5-3 grown at 1.4 and $100 \mu \mathrm{mol} \mathrm{m}^{-2} \mathrm{~s}^{-1}$ of continuous red light at $27^{\circ} \mathrm{C}$. Data normalized to WT expression in dark. Error bars represents s.e.m., $n=3$. (c) Hypocotyl measurements of WT grown for 7 days without 1-NNaphthylphthalamic acid (NPA), with $1 \mu \mathrm{M} \mathrm{NPA}$ or $50 \mu \mathrm{M} \mathrm{NPA}$ at $17^{\circ} \mathrm{C}$ or $27^{\circ} \mathrm{C}$ at indicated fluence rate of red light. Sample number $>28$, error bars represents s.e.m. (d) Transcript levels of IAA19 and IAA29 relative to IPP2 in 6-day-old WT and hy5-215 seedlings grown in 1.4 or $40 \mu \mathrm{mol} \mathrm{m}^{-2} \mathrm{~s}^{-1}$ of continuous red light at indicated temperature. Error bars represents s.e.m., $n=3$.

\section{Discussion}

In this study, we used the simple hypocotyl system to interrogate the interaction between light and temperature through the phyBPIF signalling module. phyB inhibits while PIFs promote hypocotyl extension and control is achieved through reciprocal phyB-PIF cross-regulation. Combining mathematical modelling and experimental approaches allowed us to test current assumptions on light and temperature signalling and generate nonintuitive predictions. We demonstrated that at $17^{\circ} \mathrm{C}$, phyB action was not restricted to the low fluence rate range, but continued to suppress hypocotyl elongation even at relatively high fluence rates $\left(>100 \mu \mathrm{mol} \mathrm{m}^{-2} \mathrm{~s}^{-1}\right)$. The development of model I exposed a gap in our understanding of how this is achieved. Data combined with model simulation predicted that this regulation could be achieved by ' $\mathrm{X}$ ', a moderator of PIF activity that was fluence rate and possibly temperature dependent (model II). HY5 fulfilled the requirements for $\mathrm{X}$, validating this prediction. A recent study has since shown that HY5 does indeed moderate PIF action by colocalizing to G-box elements of common gene targets ${ }^{26,27}$. Interestingly, our data show that HY5 does not appear to control
PIF activity at very low fluence rates (for example, $1.4 \mu \mathrm{mol} \mathrm{m}{ }^{-2} \mathrm{~s}^{-1}$ ), rather, it becomes increasingly dominant at higher fluence rates when HY5 protein levels increase. HY5 has previously been implicated in cold acclimation and induction of anthocyanin accumulation in response to low temperatures $\left(4^{\circ} \mathrm{C}\right)^{28,29,39}$. Here we show that HY5 is a more potent suppressor of hypocotyl elongation and PIF-controlled gene expression at the higher temperature of $17^{\circ} \mathrm{C}$. However, this control is markedly reduced at $27^{\circ} \mathrm{C}$.

Unexpectedly, we found that the 'classical' hypocotyl fluence rate response is completely temperature dependent. At cooler temperatures, red light strongly inhibits hypocotyl extension, but at $27^{\circ} \mathrm{C}$, over a minimal fluence rate, light promotes elongation. We showed that the warm temperature behaviour does not simply arise from alterations in phyB or PIF levels. Rather this effect results from a light-dependent alteration in PIF activity. Interestingly, this light-mediated control is executed through a specific gene subset that includes auxin response genes. Our data therefore offer a plausible solution to the long standing, but unexplained observation that light is required for auxin 
promotion of hypocotyl elongation at high temperatures ${ }^{6,8}$. Until now, this finding appeared to be at odds with the well-established repressive role of light in this response.

To gain an understanding of how light and temperature convergence changes the signalling characteristics of the phyBPIF module, we developed a photothermal model (Model III). This model, which incorporated temperature-dependent parameters, was able to match the physiological data by including the temperature-activated components X and Y. However, Model III could only simulate the temperature-induced switch from hypocotyl inhibition to elongation when these variables were also fluence rate dependent, suggesting a requirement for continuous irradiation. Indeed, when simulating pulsed light conditions, the model could no longer match the data. We tested this prediction by supplying seedlings with the same total fluence of red light as either continuous irradiation or 5- or 15-min discrete pulses. While we saw a minor impact of the pulse treatment at $17^{\circ} \mathrm{C}$, we could not generate the switch to promotion at $27^{\circ} \mathrm{C}$. This experiment verified our model and suggested that at both temperatures, but particularly at $27^{\circ} \mathrm{C}$, continuous light is required to generate the hypocotyl response. High fluence rate control could be mediated either through a biochemical mechanism acting as a Pr to Pfr flux counter or else via a phyB-independent mechanism such as photosynthesis. Sucrose-induced hypocotyl elongation has recently been shown to be dependent on PIFs, indicating that increased photosynthesis is a plausible candidate mechanism ${ }^{40,41}$. The models can achieve the requisite fluence rate-dependent regulation in a phyB dependent or a phyB-independent manner and so does not discriminate between these two possibilities.

Our finding that light activates PIF-dependent gene expression at $27^{\circ} \mathrm{C}$ aligns with the recent report that PIF4 access to the FT promoter is facilitated by temperature-induced chromatin modification ${ }^{31}$. This is achieved through heat-induced expulsion of the H2A.Z histone variant from nucleosomes that, when present, blocks transcription. Other studies have implicated histone acetylation in light-activated gene expression ${ }^{42-44}$. Indeed, PIF3 was recently shown to repress photosynthetic gene transcription through histone deacetylase 15 (ref. 45). It is therefore possible that the dual effect of light and temperature on transcriptional activation is mediated through dynamic changes in chromatin structure and access to DNA. Alternatively, the boost in transcription could be achieved by one or more transcription factors that work cooperatively with PIFs. A recent study demonstrated that BZR1 heterodimerizes with PIF4 and is required for heat-induced hypocotyl extension ${ }^{5}$. It is, however, uncertain that BZR1 would fulfil the requirements for ' $\mathrm{Y}$ ' as light does not appear to promote BZR1 action, rather, it leads to the accumulation of the inactive, phosphorylated form of BZR1 (ref. 46). Nonetheless, the observed elevated expression of gene subset could be achieved through an analogous co-operative heterodimerization mechanism.

In the natural environment, plants have to react simultaneously to changing light and temperature cues. This study illustrates how signal integration can culminate in unforeseen molecular signalling outputs. We have shown that temperature does not affect all reactions equally; rather, different parameters have specific temperature sensitivities, which is important to deliver distinct outcomes. Our model-based approach has shown that light imposes strict control on PIFs, by enlisting regulators of PIF activity. In cool conditions, light promotion of HY5 levels is particularly important to suppress PIF action at higher fluence rates. As temperature rises, light promotes PIF activity resulting in fluence rate-dependent promotion of hypocotyl elongation. This non-intuitive switch in light-induced behaviour was predicted by mathematical models and validated experimentally.

\section{Methods}

Growth conditions. For all experiments, seeds were surface sterilized and sown on agar plates containing $1 / 2$ Murashige and Skoog media. After 3-4 days stratification at $4{ }^{\circ} \mathrm{C}$, the seeds were exposed to $2 \mathrm{~h}$ of white light at $20^{\circ} \mathrm{C}$ followed by $22 \mathrm{~h}$ incubation in darkness before moved in to the experimental conditions.

Plant material. The phyB-9, phyB-401, hy5-215, hfr1-101, pif3-3, pif4-101, pif4-2, pif5-3 double and multiple mutants as well as 35S:PIF4-HA and 35S:PIF5-HA are all in the Columbia background and have been described previously ${ }^{22,47-52}$. 35S:HY5-HA is in the Wassilewskija background ${ }^{53}$ and della4 (rgl1-1;rgl2-1; gai-6;rga-2) in Landsberg ${ }^{54}$. 35S:PIF3-LUC has been described previously ${ }^{55}$ and for creation of 35S:PIF4-LUC, PIF4 coding sequence was inserted to the 35S:PIF3-LUC pPCV812 vector as an XbaI-SmaI DNA fragment, replacing PIF3-coding sequence. PHYB:PHYB-LUC was created by replacing the Nicotiana PHYB counter parts, cloning the Arabidopsis $P H Y B$ promoter as HindIII-Bam HI and $P H Y B$ cloning region as SmaI-StuI DNA fragments into the NtPHYB:PHYB-LUC pPCV812 binary vector ${ }^{56}$.

Immunoblotting and quantitative PCR. For immunoblotting, total protein samples from seedlings grown as indicated in the main text were separated on a $10 \%$ SDS-polyacrylamide electrophoresis gel and transferred to a nitrocellulose membrane as previously described ${ }^{6}$. For protein quantification, three biologically independent experiments were analysed using anti-HA-HRP (3F10, Roche) and anti-UGPase (AGRISERA) at a dilution of 1:1,000. ImageJ software was used for PIF4-HA quantification, which was normalized to the dark sample at $17^{\circ} \mathrm{C}$. An uncropped scan corresponding to Fig. 2a is shown in Supplementary Fig. 9. For HY5-HA quantification, Metamorph (Molecular Devices) was used and normalized to a reference sample.

Quantitative PCR was performed as previously described ${ }^{6}$. In short, the samples were harvested into RNAlater (Sigma), and total RNA was extracted using RNeasy Plant Mini Kit (Qiagen). cDNA synthesis was performed using SuperScript VILO cDNA Synthesis Kit (Invitrogen). Primers used for qPCR are listed in Supplementary Table 2 .

Protein quantification using Luciferase assay. For protein quantification using Luciferase, $\sim 100 \mathrm{mg}$ of 6-day-old seedlings (PHYB:PHYB-LUC, 35S:PIF4-LUC and 35S:PIF3-LUC) were harvested into liquid nitrogen. Samples were ground in liquid nitrogen and proteins were extracted in $200 \mu \mathrm{l}$ Promega Cell Culture Lysis Reagent complemented with $50 \mu \mathrm{M}$ of MG132, MG115, ALLN, PS1 and $1 \times$ Complete protease inhibitor cocktail, EDTA-free (Roche). The samples were centrifuged at 14,000 r.p.m. for $10 \mathrm{~min}$ at $4{ }^{\circ} \mathrm{C}$ to remove debris. In total, $50 \mu \mathrm{l}$ of each sample was then added to a 96-well plate and analysed using MicroLumatPlus LB96V Microplate Luminometer with the Luciferin-based Reporter Lysis Buffer (Promega). To determine the total protein content of the samples, $50 \mu \mathrm{l}$ of each sample was first washed using the Compat-Able Protein Assay Preparation Reagent Set (Thermo) to remove the detergent according to the manufacturer's instructions. Total protein was then assayed using Pierce BCA kit (Thermo) according to the manufacturer's instructions and absorbance was measured at $562 \mathrm{~nm}$. A dilution series using bovine serum albumin was used as a reference to determine the sample protein content.

\section{References}

1. Sullivan, J. A. \& Deng, X. W. From seed to seed: the role of photoreceptors in Arabidopsis development. Dev. Biol. 260, 289-297 (2003).

2. de Lucas, M. et al. A molecular framework for light and gibberellin control of cell elongation. Nature 451, 480-484 (2008).

3. Feng, S. et al. Coordinated regulation of Arabidopsis thaliana development by light and gibberellins. Nature 451, 475-479 (2008).

4. Lau, O. S. \& Deng, X. W. Plant hormone signaling lightens up: integrators of light and hormones. Curr. Opin. Plant Biol. 13, 571-577 (2010).

5. Oh, E., Zhu, J. Y. \& Wang, Z. Y. Interaction between BZR1 and PIF4 integrates brassinosteroid and environmental responses. Nat. Cell Biol. 14, 802-809 (2012).

6. Foreman, J. et al. Light receptor action is critical for maintaining plant biomass at warm ambient temperatures. Plant J. 65, 441-452 (2011).

7. Franklin, K. A. et al. Phytochrome-interacting factor 4 (PIF4) regulates auxin biosynthesis at high temperature. Proc. Natl Acad. Sci. USA 108, 20231-20235 (2011).

8. Gray, W. M., Ostin, A., Sandberg, G., Romano, C. P. \& Estelle, M. High temperature promotes auxin-mediated hypocotyl elongation in Arabidopsis. Proc. Natl Acad. Sci. USA 95, 7197-7202 (1998).

9. Kumar, S. V. \& Wigge, P. A. H2A.Z-containing nucleosomes mediate the thermosensory response in Arabidopsis. Cell 140, 136-147 (2010).

10. Stavang, J. A. et al. Hormonal regulation of temperature-induced growth in Arabidopsis. Plant J. 60, 589-601 (2009).

11. Sun, J., Qi, L., Li, Y., Chu, J. \& Li, C. PIF4-mediated activation of YUCCA8 expression integrates temperature into the auxin pathway in regulating Arabidopsis hypocotyl growth. PLoS Genet. 8, e1002594 (2012).

12. Sakamoto, K. \& Nagatani, A. Nuclear localization activity of phytochrome B. Plant J. 10, 859-868 (1996). 
13. Nagy, F. \& Schafer, E. Phytochromes control photomorphogenesis by differentially regulated, interacting signaling pathways in higher plants. Annu. Rev. Plant Biol. 53, 329-355 (2002).

14. Rausenberger, J. et al. An integrative model for phytochrome B mediated photomorphogenesis: from protein dynamics to physiology. PLoS ONE 5, e10721 (2010)

15. Reed, J. W., Nagatani, A., Elich, T. D., Fagan, M. \& Chory, J. Phytochrome A and phytochrome B have overlapping but distinct functions in Arabidopsis development. Plant Physiol. 104, 1139-1149 (1994).

16. Rausenberger, J. et al. Photoconversion and nuclear trafficking cycles determine phytochrome A's response profile to far-red light. Cell 146, 813-825 (2011).

17. Al-Sady, B., Ni, W., Kircher, S., Schafer, E. \& Quail, P. H. Photoactivated phytochrome induces rapid PIF3 phosphorylation prior to proteasomemediated degradation. Mol. Cell 23, 439-446 (2006).

18. Park, E. et al. Phytochrome B inhibits binding of phytochrome-interacting factors to their target promoters. Plant J. 72, 537-546 (2012).

19. Jang, I. C., Henriques, R., Seo, H. S., Nagatani, A. \& Chua, N. H. Arabidopsis phytochrome interacting factor proteins promote phytochrome $\mathrm{B}$ polyubiquitination by COP1 E3 ligase in the nucleus. Plant Cell 22, 2370-2383 (2010).

20. Koini, M. A. et al. High temperature-mediated adaptations in plant architecture require the bHLH transcription factor PIF4. Curr. Biol. 19, 408-413 (2009).

21. Nomoto, Y. et al. A circadian clock- and PIF4-mediated double coincidence mechanism is implicated in the thermosensitive photoperiodic control of plant architectures in Arabidopsis thaliana. Plant Cell. Physiol. 53, 1965-1973 (2012).

22. Oyama, T., Shimura, Y. \& Okada, K. The Arabidopsis HY5 gene encodes a bZIP protein that regulates stimulus-induced development of root and hypocotyl. Genes Dev. 11, 2983-2995 (1997).

23. Shin, J. et al. Phytochromes promote seedling light responses by inhibiting four negatively-acting phytochrome-interacting factors. Proc. Natl Acad. Sci. USA 106, 7660-7665 (2009).

24. Osterlund, M. T., Hardtke, C. S., Wei, N. \& Deng, X. W. Targeted destabilization of HY5 during light-regulated development of Arabidopsis. Nature 405, 462-466 (2000).

25. Pacín, M., Legris, M. \& Casal, J. J. Rapid decline in nuclear COP1 abundance anticipates the stabilisation of its target HY5 in the light. Plant Physiol. 164, 1134-1138 (2014).

26. Chen, D. et al. Antagonistic basic helix-loop-helix/bZIP transcription factors form transcriptional modules that integrate light and reactive oxygen species signaling in Arabidopsis. Plant Cell 25, 1657-1673 (2013).

27. Toledo-Ortiz, G. et al. The HY5-PIF regulatory module coordinates light and temperature control of photosynthetic gene transcription. PLoS Genet. 10, e1004416 (2014)

28. Catala, R., Medina, J. \& Salinas, J. Integration of low temperature and light signaling during cold acclimation response in Arabidopsis. Proc. Natl Acad. Sci. USA 108, 16475-16480 (2011)

29. Zhang, Y., Liu, Z., Liu, R., Hao, H. \& Bi, Y. Gibberellins negatively regulate low temperature-induced anthocyanin accumulation in a HY5/HYH-dependent manner. Plant Signal. Behav. 6, 632-634 (2011).

30. Quail, P. H. et al. Phytochromes: photosensory perception and signal transduction. Science 268, 675-680 (1995).

31. Kumar, S. V. et al. Transcription factor PIF4 controls the thermosensory activation of flowering. Nature 484, 242-245 (2012).

32. Yamashino, T. et al. A Link between circadian-controlled bHLH factors and the APRR1/TOC1 quintet in Arabidopsis thaliana. Plant Cell Physiol. 44, 619-629 (2003).

33. Khanna, R. et al. The basic helix-loop-helix transcription factor PIF5 acts on ethylene biosynthesis and phytochrome signaling by distinct mechanisms. Plant Cell 19, 3915-3929 (2007).

34. Hornitschek, P., Lorrain, S., Zoete, V., Michielin, O. \& Fankhauser, C. Inhibition of the shade avoidance response by formation of non-DNA binding bHLH heterodimers. EMBO J. 28, 3893-3902 (2009).

35. Hao, Y., Oh, E., Choi, G., Liang, Z. \& Wang, Z. Y. Interactions between HLH and bHLH factors modulate light-regulated plant development. Mol. Plant 5, 688-697 (2012)

36. Fairchild, C. D., Schumaker, M. A. \& Quail, P. H. HFR1 encodes an atypical bHLH protein that acts in phytochrome A signal transduction. Genes Dev. 14, 2377-2391 (2000)

37. Fankhauser, C. \& Chory, J. RSF1, an Arabidopsis locus implicated in phytochrome A signaling. Plant Physiol. 124, 39-45 (2000).

38. Soh, M. S., Kim, Y. M., Han, S. J. \& Song, P. S. REP1, a basic helix-loop-helix protein, is required for a branch pathway of phytochrome A signaling in Arabidopsis. Plant Cell 12, 2061-2074 (2000).

39. Zhang, Y., Zheng, S., Liu, Z., Wang, L. \& Bi, Y. Both HY5 and HYH are necessary regulators for low temperature-induced anthocyanin accumulation in Arabidopsis seedlings. J. Plant Physiol. 168, 367-374 (2011).

40. Lilley, J. L., Gee, C. W., Sairanen, I., Ljung, K. \& Nemhauser, J. L. An endogenous carbon-sensing pathway triggers increased auxin flux and hypocotyl elongation. Plant Physiol. 160, 2261-2270 (2012).
41. Sairanen, I. et al. Soluble carbohydrates regulate auxin biosynthesis via PIF proteins in Arabidopsis. Plant Cell 24, 4907-4916 (2012).

42. Benhamed, M., Bertrand, C., Servet, C. \& Zhou, D. X. Arabidopsis GCN5, HD1, and TAF1/HAF2 interact to regulate histone acetylation required for lightresponsive gene expression. Plant Cell 18, 2893-2903 (2006).

43. Bertrand, C. et al. Arabidopsis HAF2 gene encoding TATA-binding protein (TBP)-associated factor TAF1, is required to integrate light signals to regulate gene expression and growth. J. Biol. Chem. 280, 1465-1473 (2005).

44. Charron, J. B., He, H., Elling, A. A. \& Deng, X. W. Dynamic landscapes of four histone modifications during deetiolation in Arabidopsis. Plant Cell 21, 3732-3748 (2009).

45. Liu, X. et al. Phytochrome interacting factor3 associates with the histone deacetylase HDA15 in repression of chlorophyll biosynthesis and photosynthesis in etiolated Arabidopsis seedlings. Plant Cell 25, 1258-1273 (2013).

46. Kim, B. et al. Darkness and gulliver $2 /$ phyB mutation decrease the abundance of phosphorylated BZR1 to activate brassinosteroid signaling in Arabidopsis. Plant J. 77, 737-747 (2014).

47. Adam, E. et al. Altered dark- and photoconversion of phytochrome B mediate extreme light sensitivity and loss of photoreversibility of the phyB-401 mutant. PLoS ONE 6, e27250 (2011).

48. Reed, J. W., Nagpal, P., Poole, D. S., Furuya, M. \& Chory, J. Mutations in the gene for the red/far-red light receptor phytochrome B alter cell elongation and physiological responses throughout Arabidopsis development. Plant Cell 5, 147-157 (1993).

49. Lorrain, S., Allen, T., Duek, P. D., Whitelam, G. C. \& Fankhauser, C. Phytochrome-mediated inhibition of shade avoidance involves degradation of growth-promoting bHLH transcription factors. Plant J. 53, 312-323 (2008).

50. Nozue, K. et al. Rhythmic growth explained by coincidence between internal and external cues. Nature 448, 358-361 (2007).

51. Leivar, P. et al. Multiple phytochrome-interacting bHLH transcription factors repress premature seedling photomorphogenesis in darkness. Curr. Biol. 18, 1815-1823 (2008).

52. Duek, P. D. \& Fankhauser, C. HFR1, a putative bHLH transcription factor, mediates both phytochrome A and cryptochrome signalling. Plant J. 34, 827-836 (2003).

53. Lee, J. et al. Analysis of transcription factor HY5 genomic binding sites revealed its hierarchical role in light regulation of development. Plant Cell 19, 731-749 (2007).

54. Josse, E. M. et al. A DELLA in disguise: SPATULA restrains the growth of the developing Arabidopsis seedling. Plant Cell 23, 1337-1351 (2011).

55. Viczian, A. et al. Functional characterization of phytochrome interacting factor 3 for the Arabidopsis thaliana circadian clockwork. Plant Cell Physiol. 46, 1591-1602 (2005).

56. Bognar, L. K. et al. The circadian clock controls the expression pattern of the circadian input photoreceptor, phytochrome B. Proc. Natl Acad. Sci. USA 96, 14652-14657 (1999).

\section{Acknowledgements}

We are grateful to Professor Ferenc Nagy and Dr András Viczián for supplying the LUC seeds. We thank Dr Lorraine Kerr, manager of the KPF facility in SynthSys, for qRTPCR support and Dr Nacho Molina for useful discussions on parameter identifiability. This work was supported by the BBSRC graduate student training award (BB/F59011/1) and the BBSRC/EPSRC SABR award ROBuST (BB/F005237/1) to K.J.H., and Scottish Universities Life Science Alliance (SULSA) awards to K.J.H. and R.G.

\section{Author contributions}

K.J.H., H.J., discovered the photothermal switch. K.J.H., H.J., H.J.J. designed the research H.J., J.F., J.R.H., K.S. performed the experiments. K.J.H., R.G. and H.J.J. built and analysed the mathematical models. K.J.H., H.J., H.J.J., R.G. analysed the data and wrote the manuscript. K.J.H., R.G. provided overall guidance on the project.

\section{Additional information}

Supplementary Information accompanies this paper at http://www.nature.com/ naturecommunications

Competing financial interests: The authors declare no competing financial interests

Reprints and permission information is available online at http://npg.nature.com/ reprintsandpermissions/

How to cite this article: Johansson, H. et al. Arabidopsis cell expansion is controlled by a photothermal switch. Nat. Commun. 5:4848 doi: 10.1038/ncomms5848 (2014).

This work is licensed under a Creative Commons Attribution 4.0 International License. The images or other third party material in this article are included in the article's Creative Commons license, unless indicated otherwise in the credit line; if the material is not included under the Creative Commons license, users will need to obtain permission from the license holder to reproduce the material. To view a copy of this license, visit http://creativecommons.org/licenses/by/4.0/ 